\title{
Allergic contact dermatitis and associated allergic dermatoses: epidemiological, allergic, and immunological characteristics
}

\author{
Xinyu $\mathrm{Cai}^{1}$, Lyudmila Smirnova², Ziyuan $\mathrm{Ma}^{2}$, Ekaterina Orlova ${ }^{2}$ \\ ${ }^{1}$ School of Labour Economics, Capital University of Economics and Business, Beijing, China \\ ${ }^{2}$ Department of Dermatology and Venereology, I. M. Sechenov First Moscow State Medical University (Sechenov University), Moscow, \\ Russia \\ Adv Dermatol Allergol 2021; XXXVIII (6): 1058-1064 \\ DOI: https://doi.org/10.5114/ada.2021.104692
}

\begin{abstract}
Introduction: Allergic contact dermatitis (ACD) constitutes a considerable part of allergic morbidity among the population, although its actual prevalence is unknown as it is often not properly diagnosed.

Aim: To determine the incidence of ACD compared to other allergic dermatoses in patients from the Russian Federation and the People's Republic of China, as well as analyse immunological parameters of ACD.

Material and methods: The study enrolled a total of 248 patients aged 17-63 years divided into two groups representing the population of the Russian Federation (122 patients) and the People's Republic of China (126 patients). The total male and female ratio in both groups was 183 (74\%) to 58 (26\%).

Results: The frequency of ACD incidence among other allergic dermatoses in the group of patients from the Russian Federation was $26.2 \%$, while that in the group of patients representing the population of the PRC amounted to $22.2 \%$. In the group of patients from the Russian Federation, positive reactions to allergens were most often observed for thiomersal (29.8\%), nickel sulfate (25.2\%), and a mixture of carbamates (20.7\%), and in the group of patients from China, for nickel sulfate (30.7\%), thiomersal (26.4\%), and a mixture of carbamates (23.8\%).

Conclusions: The incidence rate of ACD among patients with allergic dermatoses is about a quarter of cases in groups from both regions. The increased expression of defensin and IFN- $\gamma$ genes can be considered as a marker of inflammation.
\end{abstract}

Key words: allergic dermatosis, allergological parameters, epidemiology, immunological parameters.

\section{Introduction}

Allergic contact dermatitis (ACD) is an inflammatory skin disease caused by a reaction of a delayed-type hypersensitivity (DTH) or type 4. It occurs as a result of a chemical or antigen contact with the skin of a sensitized individual, i.e., those having immune cells T-lymphocytes that are specific to the substance $[1,2]$.

This disease occupies a significant place in the structure of allergic morbidity in the population, although its actual prevalence is unknown as it is often incorrectly diagnosed (as other eczemas). At the same time, the prevalence of ACD varies in different regions, as well as allergens being noticeably diverse in different geographical zones [3]. The ACD is more pervasive in industrialized countries. According to a large epidemiological study, $27 \%$ of the representative population sample in five European countries had positive reactions to at least one of the contact allergens included in the so-called "primer series" [4]. The study reports allergies to nickel, flavourings, and preservatives as the most common in these countries. Allergic reactions to chromate and $p$-phenylenediamine (PPD) are less frequent but typical for certain occupational groups [5]. In Japan and Asia, the main allergens are paraphenylenediamine, lanolin, resins, and UV-absorbents [6, 7]; in America, those are lanolin, resins, metals, latex, and surfactants [8], and in Russia, those are latex, metals, dyes, topical drugs, and preservatives $[9,10]$. ACD is twice as common in women as in men [11] and often occurs at a young age, so its prevalence at the

Address for correspondence: Lyudmila Smirnova, Department of Dermatology and Venereology, I. M. Sechenov First Moscow State Medical University (Sechenov University), Moscow 119435, Russia, phone: +79166735658; e-mail: smirnovalyu27@rambler.ru Received: 13.08.2020, accepted: 24.09.2020. 
age of 12-16 years reaches $15 \%$ [12]. Among occupational skin diseases, ACD is the most common pathology.

At present, the frequency of this pathology increased, which is associated with the introduction of new chemicals that constitute drugs, cosmetic products, medical implants, household chemicals, industrial reagents, etc.

The pathogenesis of ACD is still poorly investigated, but it is known that after contact with the skin, the allergen penetrates the stratum corneum and is absorbed by Langerhans cells [13]. These cells migrate to regional lymph nodes, and the antigens absorbed by them come into contact with T-lymphocytes [14]. Antigen-specific Tlymphocytes emerge and can invade epidermis. This process is known as the sensitization phase of allergic contact dermatitis [15]. After repeated exposure to the antigen, a phase of detection occurs. Langerhans cells containing the antigen interact with antigen-specific T-lymphocytes, which triggers the proliferation process caused by cytokines [16]. As a result, a localized inflammatory response, dermatitis, occurs [17]. The intensity of the inflammatory response depends on both the sensitization capacity of the allergen and the concentration of the existing allergen. Some allergens may cause an intense inflammatory response in very small concentrations [18].

Usually, the inflammatory process is localized in areas of skin that are more susceptible to allergens, that is, on the hands, forearms, face, and neck.

Sometimes the inflammatory process goes beyond the area of sensitizing agents capturing adjacent areas of the skin, or often disguised as other common dermatoses like toxicodermatosis, atopic dermatitis (AD), eczema, psoriasis, etc.

The diagnostics of ACD are often complicated since the process of sensitization to a certain antigen can continue over a very long time like months and sometimes years. No clinical manifestations are noted in this phase. The symptoms of ACD are often not only masked but also complement the clinical picture of other dermatoses, making the identification of the disease even more difficult.

The diagnosis of allergic contact dermatitis is usually established by a combination of anamnesis, physical examination, a clinical picture, and a positive patch-test. If further evaluation is required, a skin biopsy of the affected area is performed, which usually demonstrates spongiosis [19].

Although many of the etiological factors causing ACD are known, the determination of the connection between the inflammatory process and a specific allergen can be difficult. Therefore, special allergy tests are still of high relevance.

\section{Aim}

The purpose of this study was to establish the frequency of ACD incidence among other allergic dermatoses in groups of patients from the Russian Federation and the PRC, as well as analyse immunological parameters in patients with ACD and associated allergic dermatoses.

\section{Material and methods}

A total of 248 patients in two groups representing the population of Moscow in the Russian Federation and the population of China were included in the research. The first group consisted of 122 patients, and the second group enrolled 126 patients with allergic dermatosis. The age of patients in both groups ranged from 17 to 63 years. The first group consisted of 88 (72\%) men and 34 (28\%) women, the second group consisted of 95 (75\%) men and 31 (25\%) women. The total male to female ratio in both groups was 183 (74\%) to 58 (26\%) people, respectively.

During clinical examination, complaints of patients were recorded, anamnesis data on the course of skin disease were assessed, factors leading to exacerbation of dermatosis were determined, and concomitant diseases were assessed. Skin assessment was performed to evaluate the nature of lesions.

For diagnostics and differentiation of allergic contact dermatitis, all patients had patch tests done using application - allergic test systems "AllerTest” (Nycomed Denmark A/S), which included 24 most commonly occurring chemical carriers. The test system was used according to the manufacturer's instructions. The plate was located in the upper left part of the back at a distance of about $5 \mathrm{~cm}$ from the middle line. Allergen No. 1 should be located in the upper left corner and the plate should not be attached to the edge of the shoulder blade. Each allergen must be in close contact with the skin. The location of the two place marks on the plate should be highlighted on the skin with a medical marker. The same steps were performed for plate No. 2. The test plate was positioned on the upper right side of the patient's back so that allergen number No. 13 was located in the upper left corner. The patient should wear the AllerTest for $48 \mathrm{~h}$, avoiding ingress of moisture (water, sweat) in the area of the plates. The interpretation of the results was based on the recommendations of the International Contact Dermatitis Research Group.

All patients were tested for scarification. On the back of the forearm, the place for the skin sample was treated with $70 \%$ ethyl alcohol. Afterward, marks were highlighted on the skin at a distance of 4-5 cm from each other. Surface scratches of $0.5 \mathrm{~cm}$ in length were made with the help of disposable lancets. At the same time, sampling with histamine and physiological solution (positive and negative control) was performed similarly. In case of no response to histamine, the test was considered as negative even at available allergic pathology. Test results were estimated after 15-20 min. Testing was carried out with allergens of house dust, mites of house dust, feather pillow, pet hair, and pollen allergens.

For the study of immunological parameters in the skin, a biopsy material from the area of positive reaction in the place of AllerTest plate marks was used. Samples were taken from 20 patients (10 from each geographical group) with diagnosed ACD. Besides, a biopsy material 
was taken from 20 healthy volunteers (10 from each geographical location) for comparison. The biopsy was performed applying anaesthesia with $2 \mathrm{ml}$ of 1\% lidocaine solution. The material was taken with a $3 \mathrm{~mm}$ diameter punch-scalpel, without suturing. After the procedure, an aseptic bandage was employed. The biopsy material was tested by real-time polymerase chain reaction (PCR) method with subsequent evaluation of parameters interleukin-4 (IL-4), interferon- $\gamma$ (IFN- $\gamma$ ), and $\beta$-defensins of types 1, 103, 4, and 104 and estimation of the level of fluorescent signal in each amplification cycle.

\section{Statistical analysis}

For statistical processing of PCR results, 2- $\Delta \Delta S T$ method was used, which allowed determining the quantitative index of change in gene expression in the skin of ACD patients compared to the skin of healthy people. The results were processed in statistical program Statistics 6.0.

\section{Results}

In the group of patients representing the population of the city of Moscow, the Russian Federation, diagnosis of ACD was established in 32 (26.2\%) patients, 23 (18.9\%) patients were diagnosed with atopic dermatitis (AD), and the remaining 67 (54.9\%) patients had other allergic dermatoses such as eczema verum, psoriasis, seborrheic dermatitis, rosacea, varicose eczema, acne vulgaris, lichen planus, perioral dermatitis, and large plaque parapsoriasis. In the group of patients representing the population of the city of Beijing, China, the ACD was diagnosed in 28 (22.2\%) patients, atopic dermatitis

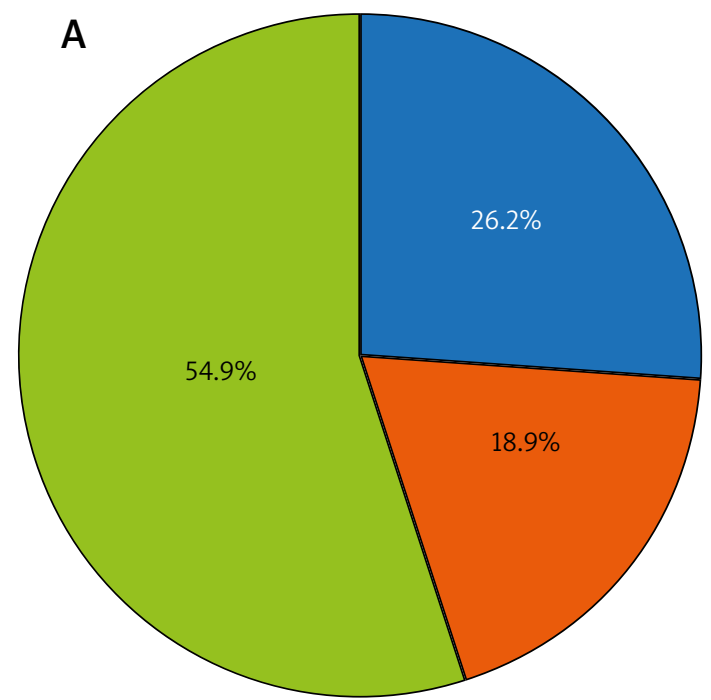

was found in 25 (19.8\%) patients, and the remaining 73 (57.9\%) patients had other allergic dermatoses (Figure 1).

All patients in both groups had a local rash, which manifested in the form of hyperaemia foci of various severity degrees, swelling, weak or moderate infiltration, lichenification, and elements filled with serous content (spotted, small papule, and vesicular) located not only in the place of skin contact with an allergen but also in skin areas remote from the triggers. In the affected area, small cracks, haemorrhagic crusts, fine-plate desquamation were observed. Patients complained of itching of weak and moderate intensity, sometimes burning in the affected area.

Anamnestic data of the investigated patients in both groups revealed that they had previously received traditional therapeutic treatment with antihistamines, vitamin preparations, and enterosorbents. External agents such as topical glucocorticosteroids (TGCS), softening and moisturizing salves/creams, and therapeutic cosmetics were used. The cases of secondary infection were treated with topical antibacterial drugs. Nevertheless, only short remissions could be achieved as a result of applied treatment.

The anamnestic data allowed identifying triggers contributing to the development of the pathology recurrence. These were, in particular, contact with an allergen - in 114 (46\%) patients, psycho-emotional stress - in 32 (13\%) patients, allergen contact in combination with psycho-emotional stress - in 18 (7\%). And, according to the data on both groups, no exacerbations were identified in 84 (34\%) patients. Out of 132 patients, in which the causative agent for the pathology exacerbation was

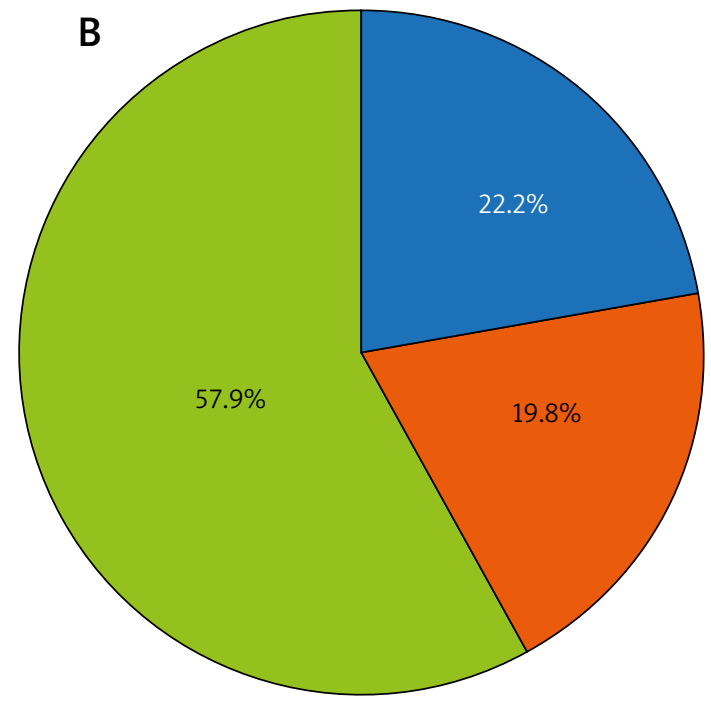

- Allergic contact dermatitis aAtopic dermatitis

- Other allergic dermatoses

Figure 1. ACD incidence rate among allergic dermatoses in groups of patients from the Russian Federation $(A)$ and the People's Republic of China (B) 
determined as contact with an allergen, 82 (62\%) people had such contact in household conditions (cosmetics, household chemicals, metal jewellery, rubber articles), and 50 (38\%) people - in production (rubbers, epoxy resin, etc.).

The localization of the inflammatory process in patients diagnosed with ACD was as follows: skin of the torso (39.4\%), forearms (35.7\%), hands (36.8\%), shin bones (22.4\%), face (27.3\%), feet (8.4\%), and neck (7.3\%). For the patients diagnosed with $A D$, inflammation was recorded on the skin of hands (73.5\%), forearms (40.4\%), torso $(31.4 \%)$, face $(12.6 \%)$, shins $(11.3 \%)$, neck $(14.8 \%)$, and feet $(6.1 \%)$. In patients with other allergic dermatoses, the localization of inflammatory processes corresponded to the clinical manifestations typical for these pathologies (true eczema on the skin of hands; psoriasis on hands, soles, and scalp; rosacea and perioral dermatitis on the face skin; seborrheic dermatitis on the skin of the face, shoulder belt, chest, interscapular region; varicose eczema on the skin of the lower leg and feet; skin itching on the skin of the neck, chest, back, abdomen, shoulders, forearms, hands, thighs, lower legs, and feet; lichen planus on the skin of shoulders, forearms, and lower leg; large plaque parapsoriasis on the skin of the back and side surfaces of the neck). The pathological process was chronic in all patients with alternating periods of exacerbation and remission.

According to the results of applying skin samples in the group of patients from the Russian Federation, positive results were most often observed for thiomersal (in $29.8 \%$ of cases), nickel sulfate (25.2\%), a mixture of carbamates (20.7\%), a mixture of thiuram derivatives (16.8\%), and potassium dichromate (14.7\%). Positive reactions were noticed to rosin, chlorine methylisothiazolinone, mercaptobenzothiazole, $p$-phenylenediamine, formaldehyde in $10.6 \%$ of cases each, to a mixture of flavouring substances and mercaptans in $8.3 \%$ of cases each, to quaternium-15 in $6.7 \%$ of cases, to lanolin, ethylenediamine dihydrochloride, and cobalt chloride in 5.8\% of cases each, to black rubber blend in $4.4 \%$ of cases, to epoxy, Peru balsam oil, $p$-butylphenol formaldehyde, and parabens in $2.7 \%$ of cases each, and neomycin sulfate in $1.2 \%$ of cases.

In the group of patients from China, positive reactions to allergens were recorded for nickel sulfate (in $30.7 \%$ of cases), thiomersal (26.4\%), a mixture of carbamates (23.8\%), a mixture of thiuram derivatives (16.8\%), p-phenylenediamine (18.3\%), potassium dichromate (12.1\%), mercaptobenzothiazole and formaldehyde (11.3\% each), rosin and chlorine methylisothiazolinone (9.4\% each), a mixture of aromatizing substances and quaternium-15 (8.7\% each), a mixture of mercaptans (7.8\%), lanolin (6.2\%), ethylenediamine dihydrochloride and cobalt chloride (5.8\%), a mixture of black rubber (5.6\%), parabens (3.2\%), epoxy, balsam of Peru, and p-tert-butyl phenol formaldehyde (2.4\% each), and neomycin sulfate (0.9\%) (Figure 2).
Thus, in the group of patients from the Russian Federation, the greatest number of positive reactions was noted for thiomersal, while that in patients from China was for nickel sulfate. Besides, in the first group, markedly more positive samples were noted for potassium chromate, rosin, chlorine methylisothiazolinone, while in the second group - for a mixture of carbamates, $p$ phenylenediamine, quarternium-15, and a mixture of black rubbers.

The results of the application tests revealed that among all investigated patients, sensitization to one allergen was observed in 91 (36.7\% of cases) patients, to two or three allergens was observed in 104 (41.9\%) patients, and to more than four allergens - in 38 (15.3\%) patients. Negative samples were recorded in 15 (6\%) patients.

A comparison of positive skin samples for allergens in $A C D$ and $A D$ patients (averaged data from both groups) is presented in Figure 3. Positive thiomersal samples were recorded with the highest frequency in patients with both pathologies (ACD in $40.5 \%$ of cases, AD in $39.2 \%$ of cases). Positive reactions to allergens for thiomersal derivatives (25.6\% of cases), nickel sulfate (22.6\%), rosin (21.2), a mixture of carbamates (14.8\%), quarternium-15 (14.6\%), a mixture of mercaptans (13.6\%), and a mixture of aromatic elements (12.4\%) were recorded with the highest frequency in patients with ACD.

In $A D$ patients, positive reactions to allergens were most often recorded for nickel sulfate (31.8\% of cases), chloromethylisothiazolinone (24.6\%), a mixture of carbamates (16\%), a mixture of flavouring substances (15.6\%),

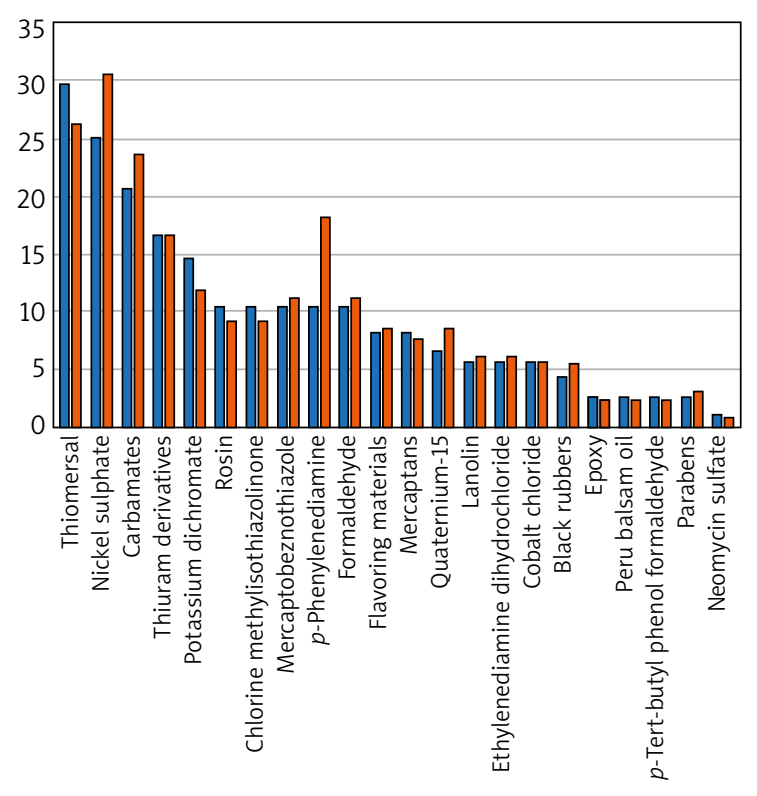

口Patients from Russia

-Patients from China

Figure 2. Comparison of positive reactions to allergens in patients with allergic dermatoses from the Russian Federation and the PRC 


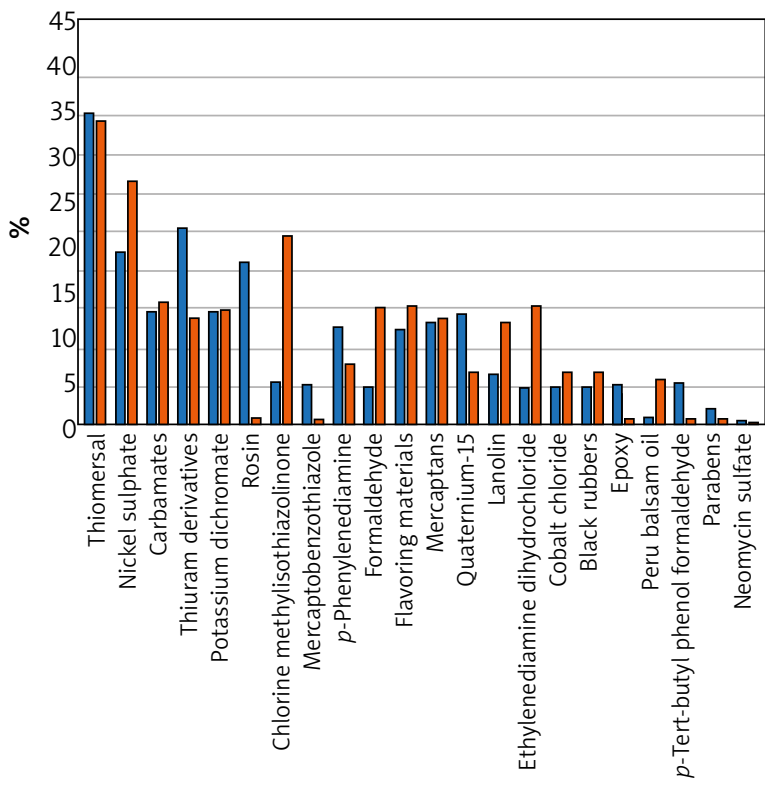

ACD patients

$\square \mathrm{AD}$ patients

Figure 3. Comparison of positive samples for allergens in patients with $A C D$ and $A D$ (averaged data from both groups)

ethylenediamine (15.4\%), formaldehyde, potassium dichromate (15\% each), a mixture of thiuram derivatives, a mixture of mercaptans (14\%), and lanolin (13.4\%).

In the application allergy testing of patients with other allergic dermatoses, a positive reaction was observed in all patients with varicose eczema, $82.3 \%$ of patients with eczema verum, $53 \%$ of patients with seborrheic dermatitis, $72 \%$ of patients with perioral dermatitis, and $83.2 \%$ of patients with psoriasis. At the same time, positive reactions to the following allergens have been established: (a) varicose eczema - lanolin, neomycin sulfate, Peru balsam oil, parabens, formaldehyde, chloromethylisothiazolinone, and a mixture of mercaptans; (b) eczema verum - epoxy, chloromethylisothiazolinone, potassium dichromate, and a mixture of carbamates and thiuram derivatives; (c) seborrheic dermatitis - p-phenylenediamine; (d) perioral dermatitis - nickel sulfate, cobalt chloride; (e) psoriasis - a mixture of carbamates, formaldehyde, and thiomersal. In general, application tests revealed positive samples in $92.6 \%$ of all patients surveyed.

Scarification allergy tests revealed the sensitization of any allergen in all AD patients. In patients with ACD, positive reactions were noted in $32.4 \%$ of cases. Positive reactions to domestic allergens ( $82.3 \%$ of cases), pollen allergens (68.6\% of cases), epidermal allergens (65.4\% of cases) prevailed in AD patients. Positive reactions to domestic allergens in $26.7 \%$ of cases, pollen allergens in $24.3 \%$ of cases, epidermal allergens in $18.8 \%$ of cases were recorded in patients with ACD.
A real-time PCR study of immunological parameters of the skin showed an increase in expression levels of defensins DEFB1, DEFB103, DEFB4, and IFN- $\gamma$ in patients with $A C D$ in comparison with healthy controls. No differences in the expression level of IL-4 and DEFB104 were noticed.

In patients with $A C D$, the increase in skin expression of the DEFB-4 gene was the highest. In $20 \%$ of patients, expression increased on average 12,000 times compared to healthy controls. The expression of the DEFB-103 gene was also significantly higher compared to healthy controls. The maximum level of expression was recorded in 34.8\% of patients in the study sample (expression increased 40 times on average). The level of DEFB gene-1 expression on the skin of patients slightly exceeded its level in healthy controls. In $27 \%$ of patients, a more than 100 -fold increase of IFN- $\gamma$-gene expression on the skin was noted. In other cases, the gene expression was increased up to 30 times in comparison with healthy controls.

\section{Discussion}

As mentioned above, the real prevalence of ACD among allergic dermatoses is difficult to determine because this pathology is often pronounced as other types of similar diseases and can occur against their background/in relation to them. Thus, a study included also patients in whom the initial diagnosis was corrected during research. For example, patients with a preliminary diagnosis of eczema verum had a final diagnosis of ACD caused by contact with substances contained in epoxy glue. Those with a preliminary diagnosis of varicose eczema eventually had ACD caused by contact with substances contained in cosmetic and external medicines. A pre-diagnosed seborrheic dermatitis resulted in ACD caused by contact with p-phenylenediamine contained in hair dye, and for preliminary diagnosis of perioral dermatitis, an ACD caused by contact with nickel sulfate contained in piercing items was recorded. In general, the studies performed showed that the incidence of ACD among other allergic dermatoses is very significant in both groups of patients examined. In the group of patients from the Russian Federation, that was close to the indicators obtained through a large epidemiological study in five European countries [4] accounting for $26.2 \%$ and $27 \%$ of allergic dermatoses, respectively. In the group of patients from China, this indicator was slightly lower (22.2\%).

The treatment of patients with contact dermatitis is based on the reduction of an inflammatory response, which is triggered by a hypersensitivity reaction of type 4 . Allergen detection and removal is the most effective treatment [20, 21]. However, it is difficult to detect sensitization to a certain allergen because this process takes a certain, sometimes rather long time and it is difficult to identify in contact with which substances the patient was in this period. And, allergens provoking ACD, are usually macromolecules (haptens), which and can trigger a sensitization process even at micro doses [13]. 
The timely diagnosis of ACD and the discovery of the provoking allergen is extremely important as the pathology worsens over time, taking on more severe forms. It is advisable to perform a comprehensive clinical and allergic examination, including patch testing as part of diagnostic workup. Patch testing is the gold standard in the diagnosis of allergic dermatoses [14] and can help to identify the allergen and avoid its effects in the future.

This study showed that among allergic dermatosis cases, the most positive sampling in patients from the Russian Federation and the second number in patients from the PRC was for thiomersal. This allergen was the leader in the number of positive reactions to allergens in patients with $A C D$ and $A D$, as well as in patients with psoriasis. It is used as a preservative in vaccines, immunoglobulin preparations, antidotes, ophthalmologic, and nasal preparations, as well as in tattoo ink [22]. The use of thiomersal as a preservative for vaccines has been contested, but now a scientific consensus insists on no conclusive evidence to support these concerns [23]. Nevertheless, research has shown high allergenicity of the substance in the progression of ACD and AD.

Patients with allergic dermatosis from China had the largest number of positive reactions to nickel sulfate, while patients from Russia had the second largest number of positive reactions to nickel sulfate after thiomersal. This allergen occupied one of the dominant positions in AD patients by the number of positive samples and, a little less, in ACD patients. Nickel compounds are among the major contact allergens worldwide, and their use in consumer products has been restricted in the European Union (EU) since 1994. However, nickel sensitization remains high [24] since a significant number of people are still exposed to nickel in their working environment. Nickel alloys are also used in the manufacture of jewellery, and another source of exposure to nickel is cell phones [25].

High frequency of positive reactions is noted for carbamates (urethanes) as polyurethanes are widely used in the production of polyurethane rubbers, adhesives, varnishes, and fibres; thiuram derivatives find application as an accelerator and stabilizer in the rubber industry and as a fungicide for the dry dressing of seeds, and potassium dichromate is used in the production of colouring and tanning of leather.

Noteworthy is a significant number of positive samples for some compounds used in the manufacture of cosmetics such as p-phenylenediamine, chloromethylisothiazolinone, formaldehyde, and a mixture of flavouring agents.

The prevalence of contact allergy is increasing worldwide. This is due to the constantly expanding range of chemicals used in the production of consumer goods. Also, significant risks of developing this pathology in contact with industrial allergens still exist. This leads to high expenses for health care systems and the economy, as well as to deterioration in the quality of life of patients.
Thus, research on allergic contact dermatosis remains of high relevance.

\section{Conclusions}

The incidence rate of ACD among patients with diagnosed allergic dermatosis was significant in the groups from both regions, and amounted to a quarter of cases, namely, $26.2 \%$ for the group of patients from the Russian Federation, and $22.2 \%$ for the group of patients from the PRC.

Mechanisms of contact hypersensitivity contribute to the development of various dermatoses. In general, tests for allergic dermatosis were positive in $92.6 \%$ of all patients examined.

Positive reactions to allergens in both groups were most frequently observed for thiomersal, nickel sulfate, a mixture of carbamates, a mixture of thiuram derivatives, and potassium dichromate.

A significant increase in the expression of DEFB-103 and DEFB-4 genes confirms the presence of defensins in the inflammation progression in ACD. The increase in expression of IFN- $\gamma$ gene testifies to its participation in delayed-type hypersensitivity in the pathogenesis of ACD. Increased expression of defensin and IFN- $\gamma$ genes may be considered as a marker of inflammation.

\section{Conflict of interest}

The authors declare no conflict of interest.

\section{References}

1. Luckett-Chastain LR, Gipson JR, Gillaspy AF, Gallucci RM. Transcriptional profiling of irritant contact dermatitis (ICD) in a mouse model identifies specific patterns of gene expression and immune-regulation. Toxicology 2018; 410: 1-9.

2. Nguyen HL, Yiannias JA. Contact dermatitis to medications and skin products. Clin Rev Allergy Immunol 2019; 56: 41-59.

3. Lazzarini R, Mendonça RF, Hafner MFS. Allergic contact dermatitis to shoes: contribution of a specific series to the diagnosis. An Bras Dermatol 2018; 93: 696-700.

4. Diepgen TL, Ofenloch RF, Bruze M, et al. Prevalence of contact allergy in the general population in different European regions. Br I Dermatol 2016; 174: 319-29.

5. Peiser M, Tralau T, Heidler J, et al. Allergic contact dermatitis: epidemiology, molecular mechanisms. Cell Mol Life Sci 2012; 69: 763-81.

6. Minamoto K. Allergic contact dermatitis from two-component acrylic resin in a manicurist and a dental hygienist. J Occup Health 2014; 56: 229-34.

7. Gupta D, Thappa DM. Dermatoses due to Indian cultural practices. Indian J Dermatol 2015; 60: 3-12.

8. Nassau S, Fonacier L. Allergic contact dermatitis. Med Clin North Am 2020; 104: 61-76.

9. Shishalova TN, Ukhanova OP. Major risk factors for development contact allergic dermatitis in children. Allergol Immunol 2011; 12: 226.

10. Shishalova TN, Ukhanova OP, Kopylov AP, et al. Pathomorphology of allergic contact dermatitis under the influence of 
low molecular weight allergens (dumbbells). Med Bulletin North Caucasus 2012; 2: 15-9.

11. Thyssen JP, Linneberg A, Menne T, Johansen JD. The epidemiology of contact allergy in the general population - prevalence and main findings. Contact Dermat 2007; 57: 287-99.

12. Özçelik S, Kulaç I, Yazıcı M, Öcal E. Distribution of childhood skin diseases according to age and gender, a single institution experience. Turk Pediatri Ars 2018; 53: 105-12.

13. Simonsen AB, Foss-Skiftesvik MH, Thyssen JP, et al. Contact allergy in Danish children: current trends. Contact Derm 2018; 79: 295-302.

14. Ameri AH, Moradi Tuchayi S, Zaalberg A, et al. IL-33/regulatory $T$ cell axis triggers the development of a tumor-promoting immune environment in chronic inflammation. Proc Nat Acad Sci USA 2019; 116: 2646-51.

15. Bil W, van der Bent SAS, Spiekstra SW, et al. Comparison of the skin sensitization potential of 3 red and 2 black tattoo inks using interleukin-18 as a biomarker in a reconstructed human skin model. Contact Derm 2018; 79: 336-45.

16. Bock S, Said A, Müller G, et al. Characterization of reconstructed human skin containing Langerhans cells to monitor molecular events in skin sensitization. Toxicol In Vitro 2018; 46: 77-85.

17. Jaulent C, Dereure O, Raison-Peyron N. Contact dermatitis caused by polyacrylamide/C13-4 isoparaffin/laureth-7 mix in an emollient cream for atopic skin. Contact Derm 2019; 81: 70-1.

18. Signore RJ. Prevention of poison ivy dermatitis with oral homeopathic Rhus toxicodendron. Dermatol Online J 2017; 23: 17.

19. Esser PR, Martin SF. Pathomechanisms of contact sensitization. Curr Allergy Asthma Rep 2017; 17: 83.

20. Choi FD, Juhasz MLW, Atanaskova Mesinkovska N. Topical ketoconazole: a systematic review of current dermatological applications and future developments. J Dermatolog Treat 2019; 30: 760-71.

21. Lampel HP, Powell HB. Occupational and hand dermatitis: a practical approach. Clin Rev Allergy Immunol 2019; 56: 60-71.

22. Sharpe MA, Livingston AD, Baskin DS. Thimerosal-derived ethylmercury is a mitochondrial toxin in human astrocytes: possible role of fenton chemistry in the oxidation and breakage of mtDNA. J Toxicol 2012; 2012: 373678.

23. Gołoś A, Lutyńska A. Thiomersal-containing vaccines a review of the current state of knowledge. Przegl Epidemiol 2015; 69: 159-61.

24. Schnuch A, Wolter J, Geier J, Uter W. Nickel allergy is still frequent in young German females - probably because of insufficient protection from nickel-releasing objects. Contact Dermatitis 2011; 64: 142-50.

25. Thyssen JP, Johansen JD, Zachariae C, Menné T. The outcome of dimethylglyoxime testing in a sample of cell phones in Denmark. Contact Dermatitis 2008; 59: 38-42. 